\title{
Risk of brain herniation after craniotomy with lumbar spinal drainage: a propensity score analysis
}

\author{
Yasushi Motoyama, MD, PhD, Tsukasa Nakajima, MD, Yoshiaki Takamura, MD, \\ Tsutomu Nakazawa, PhD, Daisuke Wajima, MD, PhD, Yasuhiro Takeshima, MD, PhD, \\ Ryosuke Matsuda, MD, PhD, Kentaro Tamura, MD, PhD, Shuichi Yamada, MD, PhD, \\ Hiroshi Yokota, MD, PhD, Ichiro Nakagawa, MD, PhD, Fumihiko Nishimura, MD, PhD, \\ Young-Su Park, MD, PhD, Mitsutoshi Nakamura, MD, PhD, and Hiroyuki Nakase, MD, PhD
}

Department of Neurosurgery, Nara Medical University, Kashihara, Nara, Japan

OBJECTIVE Lumbar spinal drainage (LSD) during neurosurgery can have an important effect by facilitating a smooth procedure when needed. However, LSD is quite invasive, and the pathology of brain herniation associated with LSD has become known recently. The objective of this study was to determine the risk of postoperative brain herniation after craniotomy with LSD in neurosurgery overall.

METHODS Included were 239 patients who underwent craniotomy with LSD for various types of neurological diseases between January 2007 and December 2016. The authors performed propensity score matching to establish a proper control group taken from among 1424 patients who underwent craniotomy and met the inclusion criteria during the same period. The incidences of postoperative brain herniation between the patients who underwent craniotomy with LSD (group $A, n=239$ ) and the matched patients who underwent craniotomy without LSD (group B, $n=239$ ) were compared. RESULTS Brain herniation was observed in 24 patients in group $A$ and 8 patients in group $\mathrm{B}(\mathrm{OR} 3.21,95 \% \mathrm{Cl} 1.36-$ $8.46, p=0.005)$, but the rate of favorable outcomes was higher in group $A(O R 1.79,95 \% \mathrm{Cl} 1.18-2.76, p=0.005)$. Of the 24 patients, 18 had uncal herniation, 5 had central herniation, and 1 had uncal and subfalcine herniation; 8 patients with other than subarachnoid hemorrhage were included. Significant differences in the rates of deep approach (OR 5.12, $95 \% \mathrm{Cl} 1.8-14.5, \mathrm{p}=0.002)$ and temporal craniotomy (OR 10.2, 95\% Cl 2.3-44.8, $\mathrm{p}=0.002)$ were found between the 2 subgroups (those with and those without herniation) in group A. In 5 patients, brain herniation proceeded even after external decompression (ED). Cox regression analysis revealed that the risk of brain herniation related to LSD increased with ED (hazard ratio 3.326, 95\% Cl 1.491-7.422, p < 0.001). Among all 1424 patients, ED resulted in progression or deterioration of brain herniation more frequently in those who underwent LSD than it did in those who did not undergo LSD (OR 9.127, 95\% Cl 1.82-62.1, p = 0.004).

CONCLUSIONS Brain herniation downward to the tentorial hiatus is more likely to occur after craniotomy with LSD than after craniotomy without LSD. Using a deep approach and craniotomy involving the temporal areas are risk factors for brain herniation related to LSD. Additional ED would aggravate brain herniation after LSD. The risk of brain herniation after placement of a lumbar spinal drain during neurosurgery must be considered even when LSD is essential.

https://thejns.org/doi/abs/10.3171/2017.12.JNS172215

KEYWORDS brain herniation; craniotomy; external decompression; lumbar spinal drainage; surgical technique

A PPROPRIATE CSF release can provide a comfortable operative field with softened brain parenchyma and a dry subarachnoid space, which can assist with smooth and correct dissection, prevent brain contusion caused by excessive brain retraction, and widen the corridor to the surgical target, even when it is deeply located. When the CSF seems difficult to release through the surgical field or a risk of ventricular tap exists, a lumbar spinal drain is often placed before surgery. Lumbar spinal drainage (LSD) is not considered a markedly in-

ABBREVIATIONS ASD = absolute standardized difference; ED = external decompression; ICH = intracranial hypotension; LSD = lumbar spinal drainage; SAH = subarachnoid hemorrhage.

SUBMITTED September 15, 2017. ACCEPTED December 19, 2017.

INCLUDE WHEN CITING Published online June 8, 2018; DOI: 10.3171/2017.12.JNS172215. 
vasive procedure, although it can result in complications such as spinal epidural hematoma and injury of the cauda equina.,8 In any type of surgery on the brain, generally prolonged CSF leakage from a spinal dural tear can be the cause of intracranial hypotension (ICH) syndrome. Patients with ICH syndrome typically present with orthostatic headache, dizziness, nausea, and, less frequently, consciousness disturbance; in such patients, neuroimaging finds brain shift downward in the caudal direction, which is called brain-sag syndrome., ${ }^{1,3,12,16,20}$ The consciousness disturbance in patients with brain-sag syndrome is thought to be a result of compression of the mesencephalon and diencephalon herniated into the tentorial hiatus. In such situations, craniotomy could aggravate the brain shift caused by LSD, and brain herniation would likely occur after the operation, ${ }^{7}$ as has been reported especially after craniotomy for ruptured aneurysmal subarachnoid hemorrhage (SAH) ${ }^{10,11,13,14}$ We often use intraoperative LSD during various types of neurosurgical procedures other than those for SAH. However, the incidence of brain herniation after craniotomy with LSD performed to treat conditions other than SAH remains unclear. The aim of this study was to investigate the incidence of and risk factors for brain herniation after craniotomy with LSD in neurosurgery overall. Thus, we performed a retrospective cohort study, and an appropriate control group was selected using a propensity score-matching method. In addition, we discuss the clinical characteristics of brain herniation after craniotomy with LSD and possible risk factors related to brain herniation.

\section{Methods}

This study is reported based on criteria from the STROBE (Strengthening the Reporting of Observational Study in Epidemiology) statement. All research protocols were approved by the institutional review board of Nara Medical University, and the need for informed consent was waived.

\section{Study Design and Patient Selection}

In the Department of Neurosurgery of Nara Medical University between 2007 and 2016, 5746 direct surgical procedures were performed; 4322 patients who underwent a type of procedure in which LSD is never used were excluded. The remaining 1424 patients were defined as the subjects for this study. Among them, 239 patients underwent craniotomy with intraoperative LSD (group A). Next, as a proper control group, patients who underwent craniotomy without LSD for the treatment of similar types of diseases in nearly the same condition as the patients who underwent LSD were selected from the 1424 subjects using the propensity score-matching method (group B). The occurrence of brain herniation within 2 weeks after surgery and the clinical outcome at postoperative day 30 , evaluated using the modified Rankin Scale (mRS) score, ${ }^{4}$ were compared between groups A and B. In both groups $\mathrm{A}$ and $\mathrm{B}$, the postoperative day that brain herniation occurred was plotted according to the Kaplan-Meier method, and the cumulative incidences of brain herniation between the 2 groups were compared.
Furthermore, the patients in group A with brain herniation were defined as group $\mathrm{C}$, which was compared with the rest of the patients without brain herniation (group D) with regard to risk factors related to the occurrence of brain herniation (Fig. 1).

In addition, some patients experienced deterioration of brain herniation after external decompression (ED). To study the adverse effects of ED on brain herniation, these patients who underwent ED retrieved from the 1424 subjects were categorized into the group with improvement or prevention (group E) or the group with progression or deterioration (group F) of their brain herniation. The rates of LSD in these 2 groups were compared.

\section{Inclusion and Exclusion Criteria}

Before selecting control patients, patients who underwent surgery for traumatic brain injury, spontaneous intracerebral hemorrhage, or cerebral infarction in the acute stage, spinal surgery, epilepsy surgery, burr hole surgery, or transsphenoidal surgery were excluded, because these types of surgery were not accompanied by LSD; pediatric patients less than 12 years of age were excluded also. In addition, cases of vascular reconstructive surgery for moyamoya disease, including superficial temporal arterymiddle cerebral artery (STA-MCA) bypass, were not included, because none of these surgeries used intraoperative LSD. In contrast, cases of deep bypass surgery, such as STA-superior cerebellar artery anastomosis for vertebrobasilar insufficiency, occipital artery-posterior inferior cerebellar artery anastomosis for dissecting or fusiformshaped aneurysms of the vertebral artery, and high-flow bypass using a radial artery graft from the external carotid artery to the $\mathrm{M}_{2}$ portion of the MCA for complex aneurysms of the internal carotid artery or MCA, were included, because some of them used LSD.

Patients with a brain abscess treated via a transcranial approach or a malignant tumor treated with craniotomy for pathological diagnosis were not excluded, because LSD was used in some patients with such a deeply located mass. Patients who underwent LSD postoperatively were not included in group A. In contrast, patients who underwent intraoperative ventricular drainage or placement of a premounted ventriculoperitoneal shunt, a cisternal drain, or an epidural drainage tube were not excluded, because the amount of CSF released during such operations varied. During the surgical procedure, in some cases, a large amount of CSF is released by suction from the subarachnoid cistern or via a catheter placed by ventricular tap. In this study, our focus was mainly on whether the lumbar spinal drain was placed during surgery, not the amount of CSF released.

\section{Propensity Score Matching}

The aim of the propensity score analyses was to minimize potential imbalances in the distribution of potential confounders between the groups of patients who underwent craniotomy with or without LSD. Propensity score matching was performed using multiple logistic regression for all 1424 subjects who underwent craniotomy with LSD versus those who underwent craniotomy without 


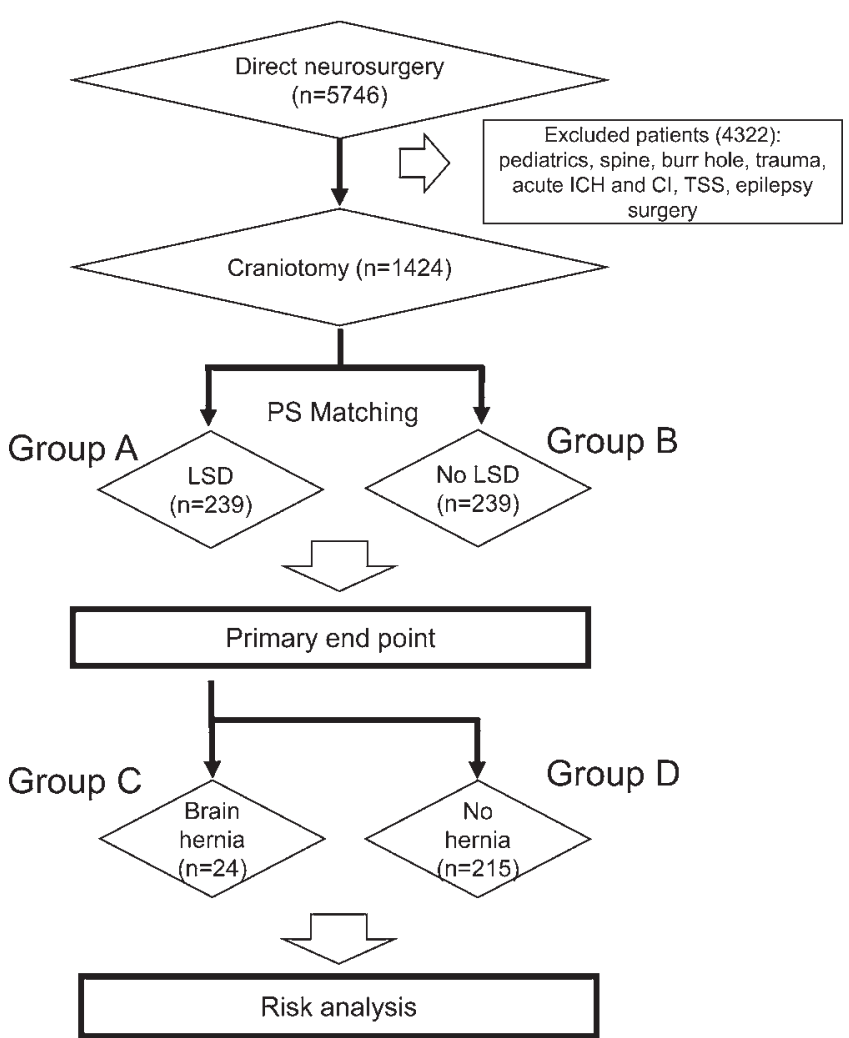

FIG. 1. Flowchart of the study population. $\mathrm{Cl}=$ cerebral infarction; $\mathrm{PS}=$ propensity score; TSS = transsphenoidal surgery.

LSD with respect to age, sex, aneurysmal SAH, presence of consciousness disturbance and focal deficits, and deep location for the surgery, all of which were defined as covariates related to the use of LSD. "Elderly" was defined as an age $\geq 70$ years. Aneurysmal SAH and use of a deep approach were adopted as covariates strongly related to the use of LSD. The interhemispheric, infratentorial supracerebellar, and subtemporal approaches and the transsylvian approach to prepontine or interpeduncular cisterns located behind the anterior circle of Willis were categorized as deep-approach procedures. The transcranial approach for extradural or extracranial lesions, including intranasal, orbital, and cavernous sinus tumors, was also categorized as a deep-approach procedure, because this approach frequently includes LSD. However, the transsylvian approach to ordinary aneurysms in the anterior circulation, the lateral suboccipital approach, and the transcortical approach were categorized as superficialapproach procedures in which LSD is rarely performed, except in patients with SAH. Consciousness disturbance and focal deficits, as indices of severity, were also adopted as covariates for matching. A level of consciousness with somnolence or more severe, or less than 4 in eye response of the Glasgow Coma Scale,${ }^{19}$ was defined as the presence of consciousness disturbance. Focal neurological deficits including hemiparesis, aphasia, and hemianopia were classified as either present or absent. All of these covariates were categorized dichotomously.

Patient matching was performed by estimated propen-
TABLE 1. Baseline characteristics of unmatched and propensity score-matched subgroups

\begin{tabular}{|c|c|c|c|c|}
\hline \multirow[b]{2}{*}{ Variable } & \multicolumn{2}{|c|}{ No. of Patients (\%) } & \multirow[b]{2}{*}{$p$ Value } & \multirow[b]{2}{*}{ ASD } \\
\hline & $\begin{array}{l}\text { Craniotomy } \\
\text { w/ LSD }\end{array}$ & $\begin{array}{l}\text { Craniotomy } \\
\text { w/o LSD }\end{array}$ & & \\
\hline \multicolumn{5}{|l|}{ Unmatched } \\
\hline Patients & 239 & 1,185 & & \\
\hline Age $>70$ yrs & $75(31.4)$ & $355(30)$ & 0.699 & 0.04 \\
\hline Female sex & $153(64)$ & $690(58.2)$ & 0.098 & 0.119 \\
\hline $\begin{array}{l}\text { Consciousness distur- } \\
\text { bance }\end{array}$ & $68(28.5)$ & $125(10.5)$ & $<0.001^{*}$ & 0.464 \\
\hline Focal deficits & $24(10)$ & $285(24.1)$ & $<0.001^{*}$ & 0.379 \\
\hline $\mathrm{SAH}$ & $123(51.5)$ & $164(13.8)$ & $<0.001^{*}$ & 0.876 \\
\hline Deep approach & $114(47.7)$ & $88(7.4)$ & $<0.001^{*}$ & 1.009 \\
\hline \multicolumn{5}{|l|}{ Propensity score matched } \\
\hline Patients & 239 & 239 & & \\
\hline Age $>70$ yrs & $75(31.4)$ & $78(32.6)$ & 0.845 & 0.027 \\
\hline Female sex & $153(64)$ & $155(64.9)$ & 0.924 & 0.071 \\
\hline $\begin{array}{l}\text { Consciousness distur- } \\
\text { bance }\end{array}$ & $68(28.5)$ & $84(35.1)$ & 0.841 & 0.144 \\
\hline Focal deficits & $24(10)$ & $24(10)$ & 1 & 0.058 \\
\hline $\mathrm{SAH}$ & $123(51.5)$ & $144(60.3)$ & 0.0653 & 0.178 \\
\hline Deep approach & $114(47.7)$ & $88(36.8)$ & 0.0205 & 0.222 \\
\hline
\end{tabular}

* Statistically significant $(p<0.05)$.

sity scores via a multiple logistic regression analysis. Using the logit estimated from the log odds of the propensity score of each patient, we matched each selected case with controls who had the nearest estimated logit value by $1: 1$ matching. Thus, group A $(n=239)$ consisted of those who underwent craniotomy with LSD, and group B $(\mathrm{n}=239)$ consisted of those who underwent craniotomy without LSD. The balance between these 2 groups for each variable was evaluated by absolute standardized differences (ASDs) before and after matching (Table 1).

\section{Clinical Outcome}

The primary end point was the occurrence of brain herniation within 2 weeks after surgery, and we compared the outcomes between patients in groups A and B. Brain herniation was diagnosed based on CT results and corresponding signs, including deterioration of consciousness disturbance, some focal signs, oculomotor palsy, respiratory distress, and decorticate or decerebrate rigidity. We ruled out other reasons for consciousness disturbance, such as symptomatic vasospasm, electrolyte imbalance, endocrinological disorder, and abnormal vital signs. Brain herniations were categorized as subfalcine, uncal, central, upward transtentorial cerebellar, or downward tonsillar based on CT and clinical findings (Table 2). Brain herniations caused by postoperative parenchymal bleeding, infarction, or subdural or epidural hematoma were all counted. However, global ischemia including the brain stem caused by respiratory failure or initial damage that resulted from severe SAH were excluded. 
TABLE 2. Brain herniation types

\begin{tabular}{|c|c|c|}
\hline Herniation Type & CT Findings & Sign (at least 1$)^{*}$ \\
\hline Subfalcine & Cingulate gyrus displaced under falx because of a shift of the cerebrum & \multirow{5}{*}{$\begin{array}{l}\text { Deterioration of con- } \\
\text { sciousness distur- } \\
\text { bance; anisocoria; eye } \\
\text { deviation; breathing ab- } \\
\text { normalities; abnormal } \\
\text { posture; decorticate or } \\
\text { decerebrate rigidity }\end{array}$} \\
\hline Uncal & Medial temporal lobe displaced medially into incisura, compressing the midbrain laterally & \\
\hline Central & $\begin{array}{l}\text { Both temporal lobes herniated into the tentorial hiatus; oblong deformity of the midbrain; } \\
\text { effacement of the perimesencephalic cistern }\end{array}$ & \\
\hline Upward transtentorial cerebellar & Cerebellum displaced up through the incisura, compressing the midbrain forward & \\
\hline Downward tonsillar & $\begin{array}{l}\text { Downward displacement of tonsil(s) to the foramen magnum, compressing medulla } \\
\text { oblongata }\end{array}$ & \\
\hline
\end{tabular}

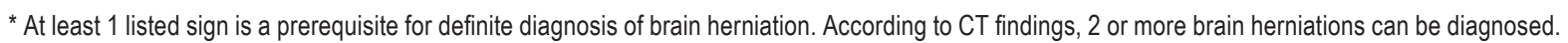

The diagnosis of symptomatic brain herniation was made by a rater (Y.M.) according to a review of the clinical and radiological courses after surgery in all patients from groups A and B. Another rater (Y.T.), who was blinded to the data about whether the patients underwent LSD, independently rated the clinical and CT findings from the same 478 patients to determine interrater reliability.

The secondary end point was a favorable outcome at postoperative day 30 , which was defined as an mRS score of less than 3.

\section{Analysis of Risk Factors and Effects of ED on Brain Herniation}

For the analysis of risk factors related to brain herniation after craniotomy with LSD, the following additional factors, along with age, sex, aneurysmal SAH, and the presence of consciousness disturbance and focal deficits, were evaluated: duration of surgery (dichotomously divided at $240 \mathrm{~min}$ ), location of the craniotomy (frontal, temporal, parietal, occipital, or suboccipital according to dichotomous evaluation for each part: transpetrosal approach including temporal and suboccipital parts or standard frontotemporal craniotomy including frontal and temporal parts in the craniotomy), and the presence of ED for controlling brain herniation, which was added at the time of or several days after the surgery. In addition, some patients experienced deterioration of brain herniation after ED. Therefore, to investigate the effect of ED on the control of brain herniation, we performed a Cox regression analysis in which the severest day of brain herniation after surgery was defined as the primary end point and the use of LSD was adopted as the explanatory variable for 478 patients from groups A and B. The risk of brain herniation caused by LSD was stratified according to whether the patient underwent ED. Last, to study the adverse effects of ED on brain herniation, the rates of craniotomy with LSD were compared between the groups that had and those that did not have an effect on brain hernia retrieved from all 1424 patients.

\section{Statistical Analysis}

All dichotomous variables are reported here as percentages. After propensity score matching, statistical comparisons between groups A and B were performed using the Fisher exact test. Interrater reliability for the occurrence of brain herniation after craniotomy was assessed using the $\kappa$ statistic. The Kaplan-Meier product limit method was used to calculate the cumulative probabilities of the incidence of brain herniation as the initial event. The cumulative incidences of brain herniation between groups A and $\mathrm{B}$ were compared with the log-rank test. To compare groups $\mathrm{C}$ and $\mathrm{D}$, each preoperative variable was subjected to univariate analysis with the Fisher exact test to determine its relationship with the occurrence of brain herniation. Variables showing significance were subsequently used in a multivariate analysis. A logistic regression model was used to identify independent risk factors from among these selected variables. To investigate the influence of ED on the risk of brain herniation after craniotomy with LSD, the incidence of brain herniation was analyzed using a Cox regression model stratified according to the use of ED. Last, for the 45 patients who underwent ED among the total 1424 subjects, the rates of LSD between groups $\mathrm{E}$ and $\mathrm{F}$ were compared using the Fisher exact test. All associations are reported as ORs with their corresponding 95\% CIs.

All statistical analyses were performed with EZR (Saitama Medical Center, Jichi Medical University), which is a graphical user interface for R (The R Foundation for Statistical Computing). More precisely, it is a modified version of $\mathrm{R}$ commander designed to add statistical functions frequently used in biostatistics. A p value $\leq 0.05$ was considered statistically significant, as long as its $95 \%$ CI did not include 1.

\section{Results}

The clinical demographics of the patients before and after propensity score matching are shown in Table 1 . Brain herniation occurred in $24(10 \%)$ patients in group $\mathrm{A}$ and in $8(3.3 \%)$ patients in group B (OR 3.21, 95\% CI $1.36-8.46, \mathrm{p}=0.005)$. In contrast, the favorable-outcome rate was higher in group A than in group B (OR 1.79, 95\% CI $1.18-2.76, \mathrm{p}=0.005$ ) (Table 3). In group $\mathrm{A}$, only $1 \mathrm{pa}-$ tient had a local complication (abscess formation at the lumbar puncture site) directly related to LSD. No other local complications, such as epidural hematoma or cauda equina injury, were seen. The interrater reliability for the occurrence of brain herniation after craniotomy was strong $(\kappa=0.948)$.

The 24 patients with brain herniation (group C) included 5 with central herniation, 18 with uncal herniation, and 1 with uncal and subfalcine herniation. No patient had 
TABLE 3. Brain herniation and favorable outcomes between groups $A$ and $B$ after propensity score matching

\begin{tabular}{|c|c|c|c|c|c|}
\hline \multirow[b]{2}{*}{ Variable } & \multicolumn{2}{|c|}{ No. of Patients (\%) } & \multirow[b]{2}{*}{ OR } & \multirow[b]{2}{*}{$95 \% \mathrm{Cl}$} & \multirow[b]{2}{*}{$\begin{array}{c}p \\
\text { Value }\end{array}$} \\
\hline & $\begin{array}{c}\text { Craniotomy } \\
\text { w/ LSD } \\
\text { (group A) }\end{array}$ & $\begin{array}{c}\text { Craniotomy } \\
\text { w/o LSD } \\
\text { (group B) }\end{array}$ & & & \\
\hline Patients & 239 & 239 & & & \\
\hline Brain herniation & $24(10)$ & $8(3.3)$ & 3.21 & $1.36-8.46$ & $0.005^{*}$ \\
\hline mRS score $<3$ & $186(77.8)$ & $158(66.1)$ & 1.79 & $1.18-2.76$ & $0.005^{*}$ \\
\hline
\end{tabular}

* Statistically significant $(p<0.05)$.

an upward transtentorial cerebellar or downward tonsillar herniation. In contrast, the 8 patients in group B with brain herniation included 2 with uncal herniation, 3 with central herniation, 1 with uncal herniation and subfalcine herniation, and 2 with upward transtentorial cerebellar and downward tonsillar herniation.

Among the 24 patients with herniation (group C), 16 patients had aneurysmal SAH, 6 had a brain tumor, 1 had an unruptured aneurysm, and 1 had vertebrobasilar insufficiency and was undergoing deep bypass surgery (Table 4). The patients with a brain tumor included 4 with meningioma located in the sphenoid ridge, petroclivus, tentorium, or falx. The remaining tumors were 1 trigeminal schwannoma and 1 recurrent metastatic pleomorphic adenoma in the cavernous sinus (Fig. 2). The $\mathrm{SAH}$ rates in groups $\mathrm{C}$ and $\mathrm{D}$ were not significantly different $(62.5 \%$ vs $50.2 \%$, respectively; OR $1.648,95 \%$ CI $0.643-4.467, p=0.287)$. The frequency of temporal craniotomy was higher in group $\mathrm{C}$ than in group $\mathrm{D}(91.7 \%$ vs $53.5 \%$, respectively; OR 9.5, 95\% CI 2.243-85.484, p $<0.001)$. The deep approach was used more frequently in group C than in group D $(79.2 \%$ vs $44.2 \%$, respectively; OR 4.771, 95\% CI 1.644-16.958, $\mathrm{p}=0.001)$. These factors related to brain herniation were not significant in group $\mathrm{B}$. The frequency of preoperative consciousness disturbance was not higher in group $\mathrm{C}$ than in group $\mathrm{D}$ ( $41.7 \%$ vs $27 \%$, respectively; OR $1.928,95 \%$ CI $0.723-$ $4.97, \mathrm{p}=0.153$ ), even though consciousness disturbance was observed more frequently in the patients with than in those without brain herniation in group B $(87.5 \%$ vs $32.5 \%$, respectively; OR $14.408,95 \%$ CI 1.799-658.046, $\mathrm{p}=0.003$ ) (Table 4). Multivariate logistic regression analysis revealed that use of a deep approach (OR 5.12, 95\% CI $1.8-14.5, \mathrm{p}=0.002$ ) and temporal craniotomy (OR 10.2, 95\% CI 2.3-44.8, $\mathrm{p}=0.002$ ) were independent risk factors for the occurrence of brain herniation after craniotomy with LSD (Table 5).

Brain herniation occurred between days 0 and 7 (Fig. 3 ). The time at which LSD was performed after surgery ranged from 0 to 15 days (median 4.5 days). Treatments for brain herniation included ED for 10 patients, internal decompression for 3, and hematoma or fluid collection evacuation for 5. Repair of an opened sinus to close the communication between the intracranial space and atmospheric air was conducted for 2 patients. Conservative treatment with the patient flat in the decubitus position and occlusion or maintaining a high LSD pressure were
TABLE 4. Demographics of the 24 patients who experienced brain herniation after craniotomy with LSD

\begin{tabular}{lc}
\hline \multicolumn{1}{c}{ Parameter } & No. of Patients (\%) \\
\hline SAH & $16(66.7)$ \\
\hline Location of aneurysm & $6(25)$ \\
\hline ICA & $4(16.7)$ \\
\hline ACA & $4(16.7)$ \\
\hline MCA & $2(8.3)$ \\
\hline Cerebrovascular disease & $1(4.2)$ \\
\hline Unruptured distal ACA aneurysm & $1(4.2)$ \\
\hline Vertebrobasilar insufficiency & $6(25)$ \\
\hline Brain tumor & $4(16.7)$ \\
\hline Meningioma & $1(4.2)$ \\
\hline Schwannoma & $1(4.2)$ \\
\hline Pleomorphic adenoma in cavernous sinus & \\
\hline Type of herniation & $5(20.8)$ \\
\hline Central & $18(75)$ \\
\hline Uncal & $1(4.2)$ \\
\hline Uncal and subfalcine & \\
\hline Signs & $24(100)$ \\
\hline Deterioration of consciousness disturbance & $3(12.5)$ \\
\hline Anisocoria & \\
\hline ACA anteior cerebra atery ICA &
\end{tabular}

$\mathrm{ACA}=$ anterior cerebral artery; ICA = internal carotid artery.

performed for 15 patients; some of the patients received more than 1 treatment. Autologous blood patches were not placed in the lumbar epidural space of any patient in this series.

The favorable outcome rate was lower in group $\mathrm{C}$ than in group D (54.2\% vs $80.5 \%$, respectively). In group C, the numbers of patients with a poor outcome (i.e., $\mathrm{mRS}$ score of $3,4,5$, or 6 ) were $6,4,0$, and 2 , respectively. The causes of death in 2 patients were brain stem injury caused by progressive herniation and pneumonia.

In group $\mathrm{C}$, progressive brain herniation with brain swelling caused by cerebral infarction could not be stopped with additional ED in 2 patients with SAH. However, in 6 patients, deterioration of brain herniation after ED was observed even though neither massive brain swelling nor intracranial hematoma formation occurred. Cox regression analysis revealed that the risk of brain herniation related to LSD increased with ED (hazard ratio 3.326, 95\% CI 1.491-7.422, p < 0.001) (Fig. 4). Given these findings in group $\mathrm{C}$, an association of $\mathrm{ED}$ with deterioration of brain herniation was suspected.

Of all 1424 patient who underwent a craniotomy, 45 patients also underwent ED. Among them, 32 showed an effect of ED with improvement or prevention of brain herniation (group E). Group F included 13 patients for whom ED failed, including those who experienced progression or deterioration in brain herniation even after ED. The rates of patients who underwent LSD were $18.8 \%$ in group $\mathrm{E}$ and $69.2 \%$ in group $\mathrm{F}$; this difference was significant (OR 9.127, 95\% CI 1.82-62.1, $\mathrm{p}=0.004$, Fisher exact test) (Fig. 5). 

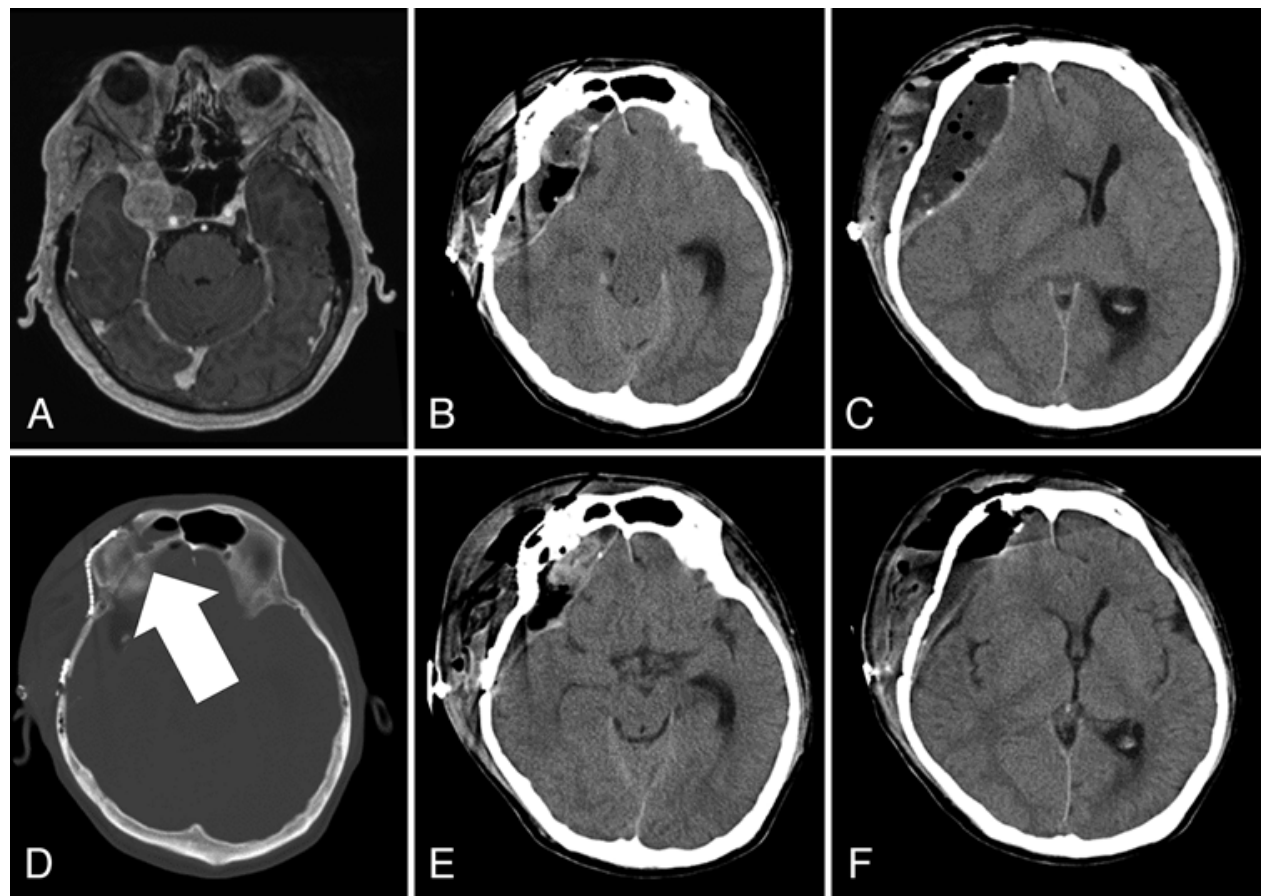

FIG. 2. CT images of a 47-year-old man with recurrence of pleomorphic adenoma in the right cavernous sinus (A) who underwent surgical intervention (a transcranial extradural approach via right frontotemporal craniotomy combined with LSD). On postoperative day 7, his consciousness decreased to stupor with restlessness. Head CT scans revealed an epidural fluid collection with air accumulation severely compressing his brain. Effacement of sulci and the perimesencephalic cisterns and right uncal herniation can be seen ( $\mathbf{B}$ and $\mathbf{C}$, respectively). A defect of the frontal sinus can also be seen on the bone target image (D, white arrow). Emergent surgery for removal of the epidural mass and repair of the opened frontal sinus was performed. CT images obtained on the day after the second surgery reveal return of brain shift and a conspicuous subarachnoid space including sulci and cisterns (E and $\mathbf{F}$, respectively). The consciousness disturbance improved immediately after surgery, and the patient was kept in the decubitus position for the following week.

\section{Discussion}

The results of this study indicate that the patients who underwent craniotomy with LSD suffered brain herniation after surgery more frequently than patients who underwent craniotomy without LSD, even though favorable clinical outcomes were more common in the first group. In general, LSD is very useful for obtaining a comfortable operative field when needed. However, brain herniation, including ICH syndrome, brain-sag syndrome, and sinking-brain syndrome, has been described after craniotomy with LSD. ${ }^{11,13}$ In patients with aneurysmal SAH, brain herniation caused by CSF hypovolemia after surgery has also been reported. ${ }^{14}$ Komotar et al.${ }^{14}$ reported that the incidence of brain herniation after clipping with LSD was 9.6\%; they also identified prolonged operative time and global cerebral edema on admission CT as risk factors. In our series, among all patients who underwent neurosurgery, including clipping for aneurysmal SAH, the incidence of brain herniation that resulted from craniotomy with LSD was $10 \%$. Meanwhile, in the subgroup of patients with SAH in our series, the incidence of brain herniation after craniotomy with LSD was $11.7 \%$, which was higher than that reported by Komotar et al., perhaps because of the inclusion in our study of not only patients with pure brain-sag syndrome without a mass lesion but also those with brain herniation with hematoma or infarction. In contrast, in patients who underwent craniotomy for something other than aneurysmal SAH, the incidence of brain herniation after craniotomy with LSD was $8.1 \%$.

The risk factors for brain herniation were investigated in a comparison of groups $\mathrm{C}$ and $\mathrm{D}$. Temporal craniotomy was performed most frequently among the patients with brain herniation after LSD. If a lesion is located in the area that requires temporal craniotomy and postoperative swelling occurs, the mass effect or brain shift could easily result in uncal herniation. It is interesting to note that in our series, parietal and occipital craniotomies were performed in only 1 patient in group $\mathrm{C}$. These areas might be less likely than the frontal and temporal areas to develop brain herniation as a result of mass effect. In group C, no patients experienced upward or downward cerebellar herniation.

Use of a deep approach was also an independent risk factor for the occurrence of brain herniation after craniotomy with LSD. It induces not only a large amount of CSF release but also destruction of the arachnoid membrane that functions in CSF regulation in a wide area or a higher risk of brain damage leading to contusion or swelling after surgery.

In contrast, the rates of those who underwent a long operation were not significantly different between groups $\mathrm{C}$ and D. CSF release was probably correlated with operation 
TABLE 5. Demographic and clinical characteristics and perioperative factors related to brain herniation

\begin{tabular}{|c|c|c|c|c|c|c|}
\hline \multirow[b]{2}{*}{ Factor } & \multicolumn{2}{|c|}{ No. of Patients (\%) } & \multicolumn{2}{|l|}{ Univariate Analysis } & \multicolumn{2}{|c|}{ Multivariate Analysis } \\
\hline & Hernia (group C) & No Hernia (group D) & OR $(95 \% \mathrm{Cl})$ & $p$ Value & OR $(95 \% \mathrm{Cl})$ & $\mathrm{p}$ Value \\
\hline \multicolumn{7}{|l|}{ Group A } \\
\hline Patients & 24 & 215 & & & & \\
\hline \multicolumn{7}{|l|}{ Preop } \\
\hline Age $>70$ yrs & $3(12.5)$ & $72(33.5)$ & $0.285(0.0527-1.001)$ & 0.0377 & & \\
\hline Female sex & $14(58.3)$ & $139(64.7)$ & $0.7664(0.3-2.028)$ & 0.6544 & & \\
\hline Consciousness disturbance & $10(41.7)$ & $58(27)$ & $1.928(0.723-4.97)$ & 0.153 & & \\
\hline Focal deficits & $2(8.3)$ & $22(10.2)$ & $0.798(0.0854-3.631)$ & 1 & & \\
\hline Deep approach & $19(79.2)$ & $95(44.2)$ & $4.771(1.644-16.958)$ & $0.001^{*}$ & $5.12(1.8-14.5)$ & $0.002^{*}$ \\
\hline $\mathrm{SAH}$ & $9(37.5)$ & $108(50.2)$ & $1.648(0.643-4.467)$ & 0.287 & & \\
\hline \multicolumn{7}{|l|}{ Involved area of craniotomy } \\
\hline Frontal & $20(83.3)$ & $155(72.1)$ & $1.931(0.611-8.09)$ & 0.332 & & \\
\hline Temporal & $22(91.7)$ & $115(53.5)$ & $9.5(2.243-85.484)$ & $<0.001^{*}$ & $10.2(2.3-44.8)$ & $0.002^{*}$ \\
\hline Parietal & $0(0)$ & $14(6.5)$ & $0(0-2.724)$ & 0.372 & & \\
\hline Occipital & $1(4.2)$ & $22(10.2)$ & $0.383(0.009-2.605)$ & 0.484 & & \\
\hline Suboccipital & $2(8.3)$ & $26(12.1)$ & $0.662(0.0714-2.964)$ & 0.7488 & & \\
\hline \multicolumn{7}{|l|}{ Postop } \\
\hline Duration of op >240 mins & $15(62.5)$ & $127(59.1)$ & $1.154(0.45-3.133)$ & 0.829 & & \\
\hline Additional ED & $10(41.7)$ & $5(2.3)$ & $28.929(7.797-123.353)$ & $<0.001^{*}$ & & \\
\hline mRS score $<3$ & $13(54.2)$ & $173(80.5)$ & $0.288(0.11-0.766)$ & $0.007^{*}$ & & \\
\hline \multicolumn{7}{|l|}{ Group B } \\
\hline Patients & 8 & 231 & & & & \\
\hline \multicolumn{7}{|l|}{ Preop } \\
\hline Age $>70$ yrs & $3(37.5)$ & $75(32.5)$ & $1.247(0.189-6.603)$ & 0.7182 & & \\
\hline Female sex & $5(62.5)$ & $150(64.9)$ & $0.9(0.17-5.945)$ & 1 & & \\
\hline Consciousness disturbance & $7(87.5)$ & $156(32.5)$ & $14.408(1.799-658.046)$ & $0.003^{*}$ & & \\
\hline Focal deficits & $2(25)$ & $21(9.1)$ & $3.30763(0.308-20.073)$ & 0.1737 & & \\
\hline Deep approach & $1(12.5)$ & $87(37.7)$ & $0.238(0.005-1.899)$ & 0.2638 & & \\
\hline $\mathrm{SAH}$ & $7(87.5)$ & $137(59.3)$ & $4.478(0.598-218.514)$ & 0.1505 & & \\
\hline \multicolumn{7}{|l|}{ Involved area of craniotomy } \\
\hline Frontal & $7(87.5)$ & $163(70.6)$ & $2.91(0.363-133.447)$ & 0.4442 & & \\
\hline Temporal & $6(75)$ & $143(61.9)$ & $1.842(0.32-19.05)$ & 0.7136 & & \\
\hline Parietal & $0(0)$ & $12(5.2)$ & $0(0-12.096)$ & 1 & & \\
\hline Occipital & $0(0)$ & $12(5.2)$ & $0(0-12.096)$ & 1 & & \\
\hline Suboccipital & $1(12.5)$ & $51(22.1)$ & $0.505(0.011-4.083)$ & 1 & & \\
\hline \multicolumn{7}{|l|}{ Postop } \\
\hline Duration of surgery $>240$ mins & $6(75)$ & $154(66.7)$ & $1.498(0.26-15.513)$ & 1 & & \\
\hline Additional ED & $6(75)$ & $11(4.8)$ & 56.651 (8.906-626.2) & $<0.001^{*}$ & & \\
\hline $\mathrm{mRS}$ score $<3$ & $0(0)$ & $158(68.4)$ & $0(0-0.281)$ & $<0.001^{*}$ & & \\
\hline
\end{tabular}

* Statistically significant $(p<0.05)$.

duration. Brain herniation did not always occur on the day immediately after the surgery. Continuous CSF release itself, but not the amount of CSF released during surgery, might be associated with the development of brain herniation.

Several recent case reports of paradoxical brain herniation after ED have been published. The cause of this type of brain herniation is thought to be exposure of the brain to atmospheric air pressure, which has been reported to be associated with lumbar tap or spontaneous ICH syndrome..$^{5,6,21}$ The mechanism of such brain herniation is thought to be similar to that in our patients with deterioration of brain herniation after ED.

In patients with brain swelling caused by parenchymal hematoma or infarction, ED would prevent increasing intracranial pressure. However, in patients who underwent LSD, ED could have an adverse effect on the clinical course after brain herniation (Fig. 6). With brain swell- 


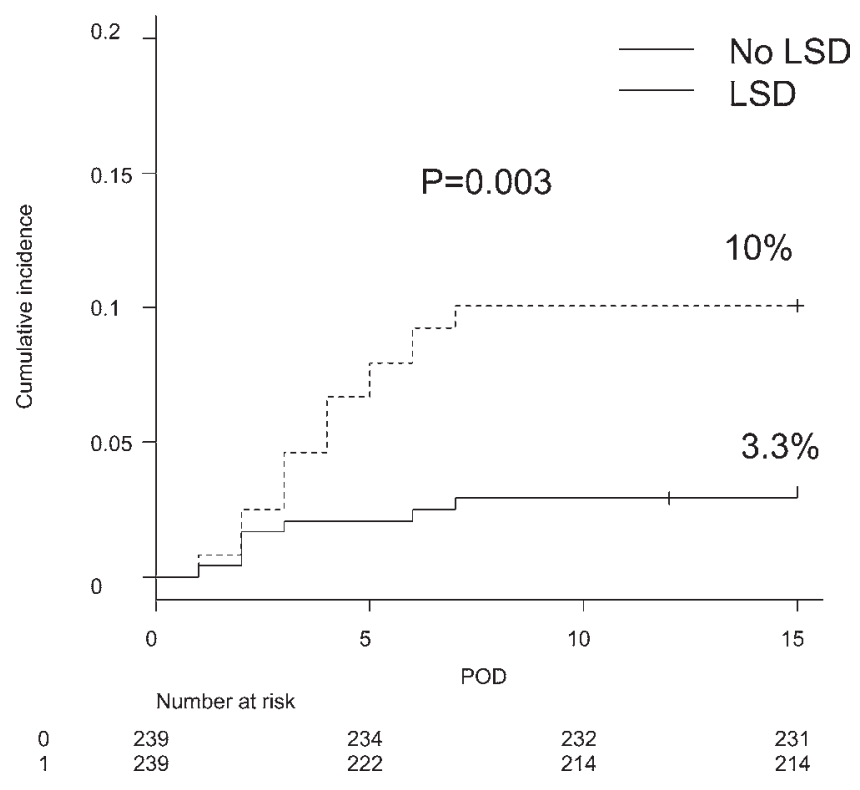

FIG. 3. Kaplan-Meier curves showing the cumulative incidence of brain herniation in groups $A$ and $B$. The log-rank test shows a significant difference $(p=0.003)$. POD = postoperative day. ing that requires ED after surgery, LSD is thought to be contraindicated. Even when a patient undergoing craniotomy with LSD shows no postoperative brain swelling, ED could paradoxically provoke deterioration of brain herniation as a result of the following mechanisms. CSF hypovolemia caused by excessive release of CSF can cause loss of buoyancy, which accelerates brain sagging and causes wedging into the tentorial hiatus. The flow of CSF through the perimesencephalic cistern is subsequently obstructed by the brain wedged into the tentorial hiatus. The loss of communication results in a pressure difference between the supratentorial and infratentorial compartments. Continuous CSF release from a lumbar spinal drain and epiarachnoid fluid collection or hematoma including air accumulation would cause an increased pressure difference that would aggravate brain herniation (Fig. 7).

With LSD, ED resulted in air entering the epidural space to make the pressure in the supratentorial space equal to atmospheric air pressure. If the pressure in the infratentorial space was lower than atmospheric air pressure, brain herniation toward the caudal direction would worsen. Likewise, the opening of an air sinus of the cranium after craniotomy with LSD is thought to be very likely to cause brain herniation, because the air accumulation would increase the pressure gradient between the supratentorial and infratentorial spaces (Fig. 7D). In such cases, the brain seems to sag and become wedged into the tentorial hiatus. Therefore, with continuous CSF drainage by a

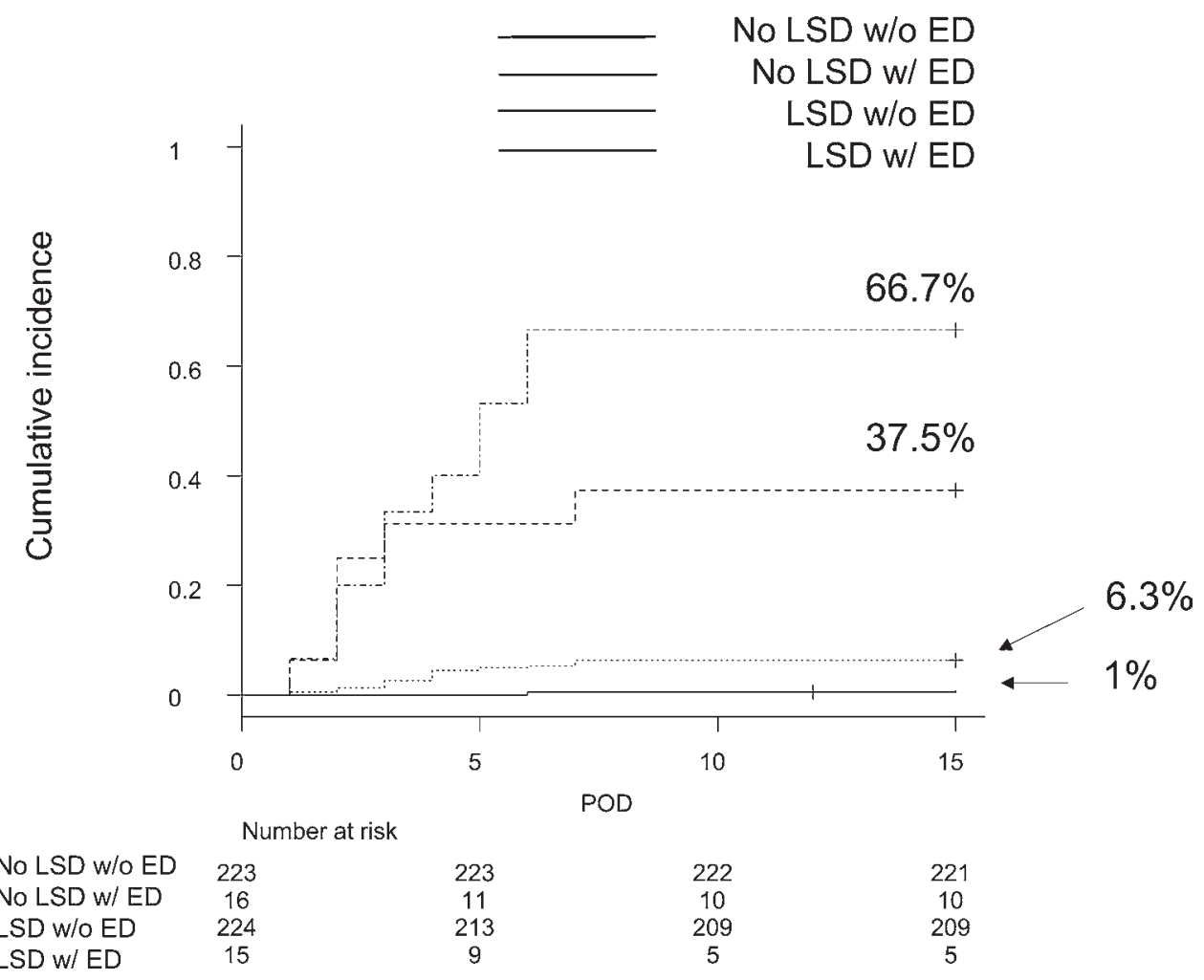

FIG. 4. Kaplan-Meier curves showing the cumulative incidence of brain herniation in groups $A$ and $B$, stratified according to the use of ED. The incidence of brain herniation related to LSD increased with additional ED. On Cox regression analysis, the hazard ratio was $3.326(95 \% \mathrm{Cl} 1.491-7.422, \mathrm{p}<0.001)$. 


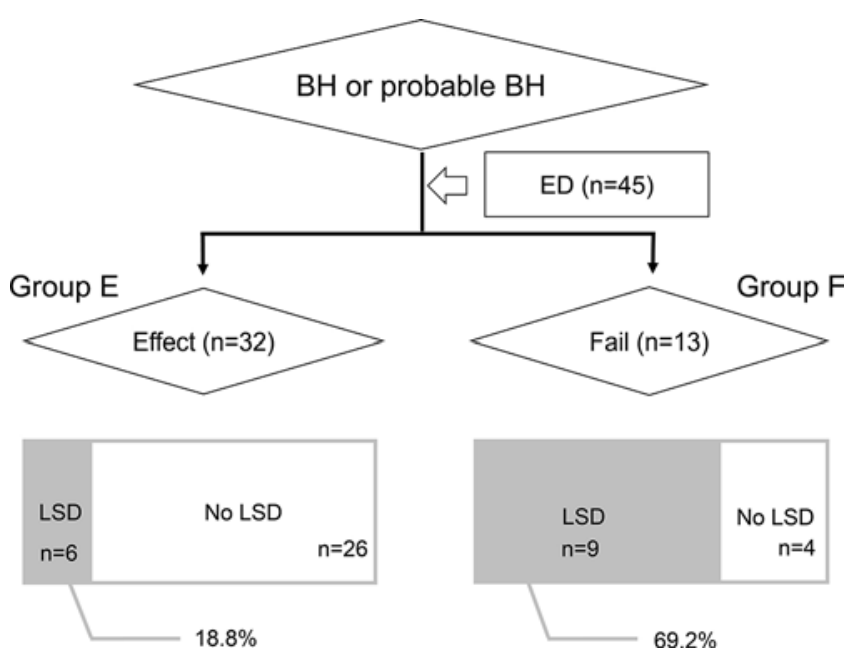

FIG. 5. Among all 1424 patients, 45 underwent ED for brain herniation $\mathrm{BH}$ or probable $\mathrm{BH}$ and were divided into a group of patients for whom ED did or a group of patients for whom ED did not have an effect on their $\mathrm{BH}$. In the group with progression or deterioration of $\mathrm{BH}$ (group F), the rate of LSD was significantly higher than it was in the group with improvement or prevention of $\mathrm{BH}$ (group E) $(69.2 \%$ vs $18.8 \%$, respectively; OR $9.127,95 \% \mathrm{Cl} 1.828-56.272, p=0.004$, Fisher exact test).

lumbar spinal drain, the elevation of head position might also be dangerous because of the suction effect caused by the accelerated loss of isotropic hydrostatic pressure in the CSF caused by gravity with ED or the opening of an air sinus.
In our series, brain herniation after craniotomy related to LSD was treated with multiple interventions, including direct surgery, maintaining the patient in the decubitus position, and occluding the drain. Surgical intervention included ED, internal decompression, hematoma or fluid collection removal, and sinus packing to repair opened air cells. Blood patch sealing of spinal dural tears was not performed in our study. In general, sealing of a dural tear by using a blood patch has been described as effective treatment for ICH syndrome. ${ }^{2}$ Some reports have indicated that blood patches improved brain herniation related to ICH syndrome. ${ }^{17,18}$ Given the frequency and pathophysiology of brain herniation related to LSD, a prophylactic blood patch should probably be placed to prevent brain herniation after craniotomy accompanied by LSD.

The Trendelenburg position has been reported to be effective for improving brain herniation. ${ }^{13,14}$ However, in symptomatic patients with an apparent mass-like hematoma or infarction, even including air accumulation and brain herniation, the Trendelenburg position alone would not be considered appropriate treatment. Surgical intervention might be necessary for an apparent mass lesion even if CSF hypovolemia caused by LSD was the initial cause of the brain herniation. In such a situation, an immediate blood patch should be added after removal of the space-occupying mass, which would provide more effective prevention of recurrence and early improvement. ${ }^{15}$

Cranioplasty has been reported to paradoxically improve brain herniation after ED. ${ }^{6}$ Closing the communication between the intracranial space and atmospheric air decreases the pressure difference between the supraten-
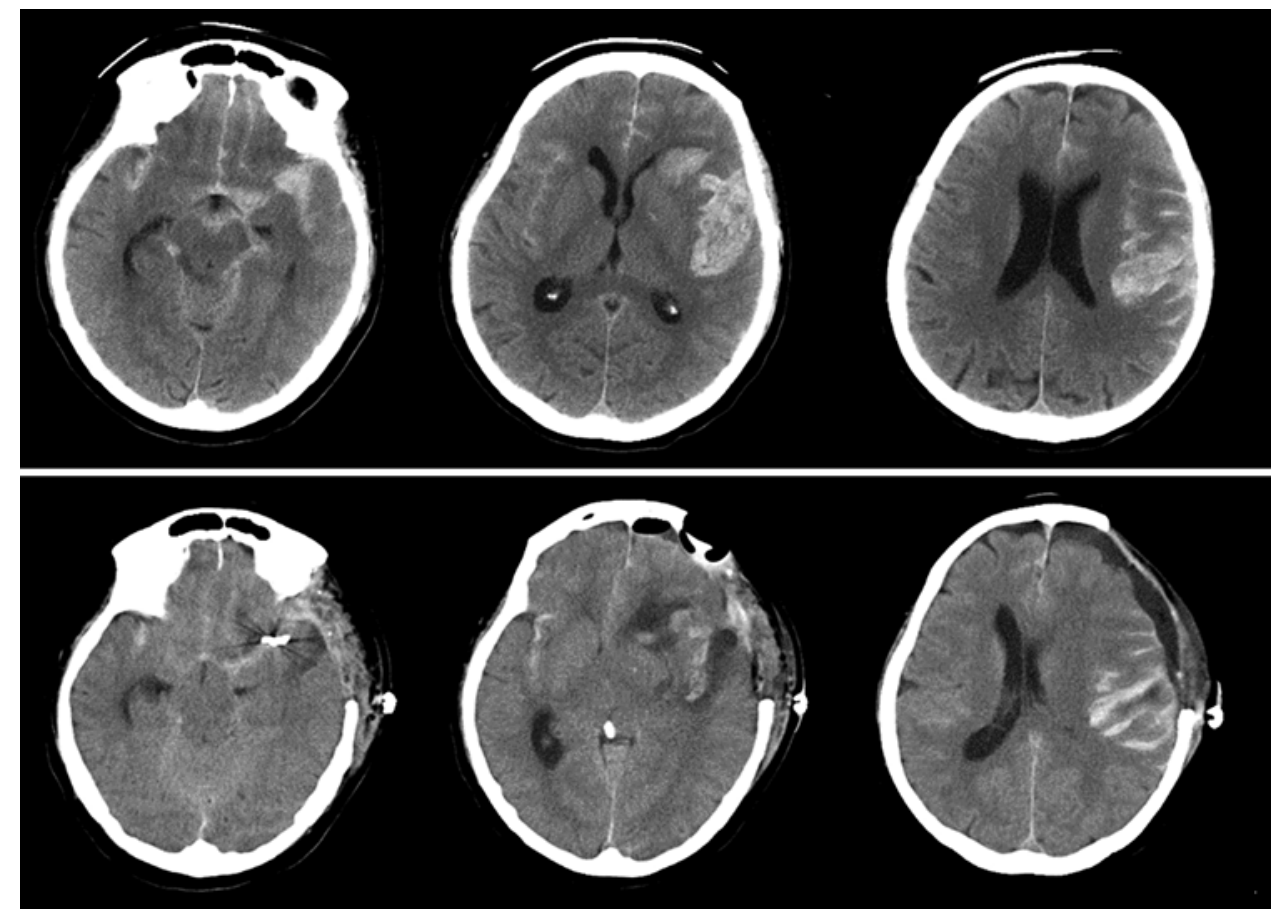

FIG. 6. Upper: A 65-year-old woman with SAH caused by a ruptured MCA aneurysm with a sylvian hematoma (Hunt and Hess grade IV $)^{9}$ underwent clipping with LSD and prophylactic ED. Lower: CT scans 4 days after surgery show brain herniation, even though bulging cannot be seen in the decompressed part. 

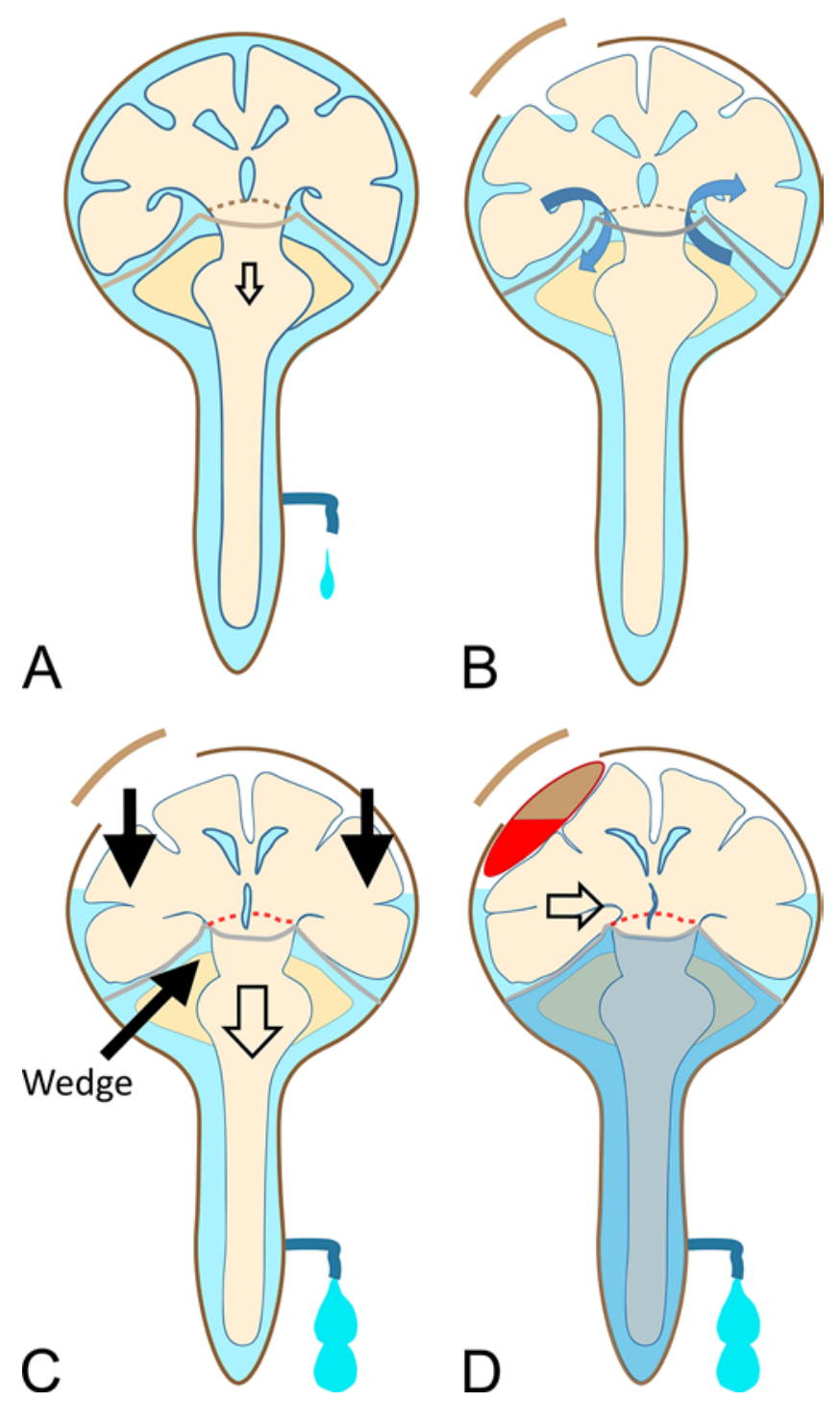

FIG. 7. Schema illustrating the skull, brain, spine, and tentorium. A: Intracranial volume composed of brain parenchyma, blood bed, and CSF, which are released by LSD and lead to brain sag. $B$ : The brain floats by buoyancy even after craniotomy. C: Craniotomy with LSD leading to CSF release, which decreases the buoyant force, wedging the brain. The wedged brain and continuous CSF release create a pressure difference between the supratentorial and infratentorial compartments. D: The epiarachnoid mass containing air aggravates brain herniation. Copyright Yasushi Motoyama. Published with permission. Figure is available in color online only.

torial and infratentorial compartments. Similarly, sinus packing is necessary and effective in the surgical treatment of brain herniation related to LSD, as shown in Fig. 4. In other words, a communication with atmospheric air through the opening of an air sinus of the cranium would be likely to prompt brain herniation after craniotomy with LSD.

In propensity matching, ASD did not reach a value less than 0.1 for some covariates. Imbalance of the cohort because of the limited number of patients cannot be ruled out and could have affected the analysis. The number of patients with brain herniation associated with LSD was too small to statistically evaluate related risk factors, even though it is not an extremely rare complication. Therefore, by comparing risk factors between groups $\mathrm{A}$ and $\mathrm{B}$, clinically significant factors were identified. However, confounding factors related to brain herniation could not be excluded completely. Furthermore, multivariate analysis using a large number of cases might be necessary to study the risk factors in detail.

In this study, favorable clinical outcomes were more common in group A than in group B. However, the result of this secondary outcome should be interpreted carefully because of selection bias based on the severity of the enrolled patients' condition. In our study, the presence of consciousness disturbance was adopted as a covariate to obtain a well-balanced cohort using propensity score matching. However, in patients with severe SAH, for example, when accompanied by intraventricular hemorrhage or acute hydrocephalus, ventricular drainage tended to be used instead of LSD. Therefore, there might have been fewer patients with a severe condition in group $A$ than in group B when they were allocated initially. As a result, the clinical outcomes might have been better in group A than in group B.

Strictly speaking, the correct diagnosis of tonsillar herniation would require sagittal MRI. In our series, we observed 2 patients in group B with upward transtentorial cerebellar and tonsillar herniation in whom diffuse swelling of the whole cerebellum as a result of ischemic insult after surgery for severe SAH caused by vertebral artery dissection herniated into not only the tentorial hiatus but also the foramen magnum. These herniations were diagnosed based on not only CT findings but also corresponding clinical signs, including respiratory failure, decerebrate rigidity, and cardiac arrest. Other than these 2 cases, however, we did not find any cases of cerebellar herniation into the foramen magnum suspected among the patients in either group A and B with some clinically neurological deterioration.

This study was retrospective and observational. Treatment for brain herniation related to craniotomy with LSD requires a comparative study that includes blood patching to evaluate the therapeutic effect appropriately.

\section{Conclusions}

When LSD is performed during craniotomy, brain herniation is more likely to occur after surgery, even though the patient's clinical outcome might be better after LSD. The use of a deep approach and temporal craniotomy are risk factors for brain herniation related to LSD. Brain herniation after LSD can occur after craniotomy to treat conditions other than SAH, even though parietal and occipital craniotomies rarely caused herniation. Brain herniation after craniotomy with LSD is not always observed in severely ill patients with consciousness disturbance, and continuous CSF release and an additional mass, including air collection in the epiarachnoid space of the supratentorial area, increase the risk of brain herniation. Additional ED would aggravate brain herniation with LSD. Therefore, in the setting of possible brain herniation that requires ED, 
LSD should be avoided even if the mass lesion is to be removed. When considering placement of a lumbar spinal drain during neurosurgery, the risk of brain herniation should be taken into account, even when LSD is essential.

\section{Acknowledgments}

We acknowledge and thank Takashi Inoue, MPH (Institute for Clinical Translational Science, Nara Medical University), for his help with statistical analysis and Aki Shimizu, Nao Sato, Haruna Tamaru, Misa Yang, Tomotaka Yamaguchi, and Ayano Ohzawa (medical students at Nara Medical University) for being in charge of collecting data from medical records.

\section{References}

1. Alaraj A, Munson T, Herrera SR, Aletich V, Charbel FT, Amin-Hanjani S: Angiographic features of "brain sag". J Neurosurg 115:586-591, 2011

2. Benzon HT, Nemickas R, Molloy RE, Ahmad S, Melen O, Cohen B: Lumbar and thoracic epidural blood injections to treat spontaneous intracranial hypotension. Anesthesiology 85:920-922, 1996

3. Bloch J, Regli L: Brain stem and cerebellar dysfunction after lumbar spinal fluid drainage: case report. J Neurol Neurosurg Psychiatry 74:992-994, 2003

4. Bonita R, Beaglehole R: Recovery of motor function after stroke. Stroke 19:1947-1500, 1988

5. Creutzfeldt CJ, Vilela MD, Longstreth WT Jr: Paradoxical herniation after decompressive craniectomy provoked by lumbar puncture or ventriculoperitoneal shunting. J Neurosurg 123:1170-1175, 2015

6. Díaz-Romero R, Avendaño P, Coloma G: Life-threatening paradoxical brain herniation rapidly reversed by emergency cranioplasty repair: a case report. Acta Neurochir (Wien) 157:2031-2032, 2015

7. Domenicucci M, Mancarella C, Santoro G, Dugoni DE, Ramieri A, Arezzo MF, et al: Spinal epidural hematomas: personal experience and literature review of more than 1000 cases. J Neurosurg Spine 27:198-208, 2017

8. Hulou MM, Abd-El-Barr MM, Gormley WB, Zamani AA, Dunn IF, Al-Mefty O: The frequency and severity of intracranial hypotension post-intraoperative lumbar drainage using a Tuohy needle and the traditional needle. Br J Neurosurg 30:438-443, 2016

9. Hunt WE, Hess RM: Surgical risk as related to time of intervention in the repair of intracranial aneurysms. J Neurosurg 28:14-20, 1968

10. Kawahara I, Tsutsumi K, Matsunaga Y, Takahata H, Ono T, Toda K, et al: Early awareness of cerebrospinal fluid hypovolemia after craniotomy for microsurgical aneurysmal clipping. Acta Neurochir (Wien) 155:1543-1548, 2013

11. Kelley GR, Johnson PL: Sinking brain syndrome: craniotomy can precipitate brainstem herniation in CSF hypovolemia. Neurology 62:157, 2004

12. Kestle JR, Walker ML: A multicenter prospective cohort study of the Strata valve for the management of hydrocepha- lus in pediatric patients. J Neurosurg 102 (2 Suppl):141-145, 2005

13. Komotar RJ, Mocco J, Ransom ER, Mack WJ, Zacharia BE, Wilson DA, et al: Herniation secondary to critical postcraniotomy cerebrospinal fluid hypovolemia. Neurosurgery 57:286-292, 2005

14. Komotar RJ, Ransom ER, Mocco J, Zacharia BE, McKhann GM II, Mayer SA, et al: Critical postcraniotomy cerebrospinal fluid hypovolemia: risk factors and outcome analysis. Neurosurgery 59:284-290, 2006

15. Loya JJ, Mindea SA, Yu H, Venkatasubramanian C, Chang SD, Burns TC: Intracranial hypotension producing reversible coma: a systematic review, including three new cases. J Neurosurg 117:615-628, 2012

16. Mokri B: Spontaneous cerebrospinal fluid leaks: from intracranial hypotension to cerebrospinal fluid hypovolemia-evolution of a concept. Mayo Clin Proc 74:1113-1123, 1999

17. Muehlschlegel S, Voetsch B, Sorond FA: Emergent epidural blood patch: lifesaving treatment of paradoxical herniation. Arch Neurol 66:670-671, 2009

18. Samadani U, Huang JH, Baranov D, Zager EL, Grady MS: Intracranial hypotension after intraoperative lumbar cerebrospinal fluid drainage. Neurosurgery 52:148-152, 2003

19. Teasdale G, Jennett B: Assessment of coma and impaired consciousness. A practical scale. Lancet 304:81-84, 1974

20. Yoon MK, Parsa AT, Horton JC: Skull thickening, paranasal sinus expansion, and sella turcica shrinkage from chronic intracranial hypotension. J Neurosurg Pediatr 11:667-672, 2013

21. Zhao J, Li G, Zhang Y, Zhu X, Hou K: Sinking skin flap syndrome and paradoxical herniation secondary to lumbar drainage. Clin Neurol Neurosurg 133:6-10, 2015

\section{Disclosures}

The authors report no conflict of interest concerning the materials or methods used in this study or the findings specified in this paper.

\section{Author Contributions}

Conception and design: Motoyama, Takeshima. Acquisition of data: Motoyama, Nakajima. Analysis and interpretation of data: Motoyama, Takamura, Nakase. Drafting the article: Motoyama, Nakajima. Critically revising the article: Motoyama, Nakazawa, Wajima, Takeshima, Matsuda, Tamura, Yamada, Yokota, Nakagawa, Nishimura, Park, Nakamura, Nakase. Reviewed submitted version of manuscript: Motoyama, Takamura, Nakazawa, Wajima, Takeshima, Matsuda, Tamura, Yamada, Yokota, Nakagawa, Nishimura, Park, Nakamura, Nakase. Approved the final version of the manuscript on behalf of all authors: Motoyama. Statistical analysis: Motoyama. Study supervision: Nakase.

\section{Correspondence}

Yasushi Motoyama: Nara Medical University, Nara, Japan. myasushi@naramed-u.ac.jp. 